
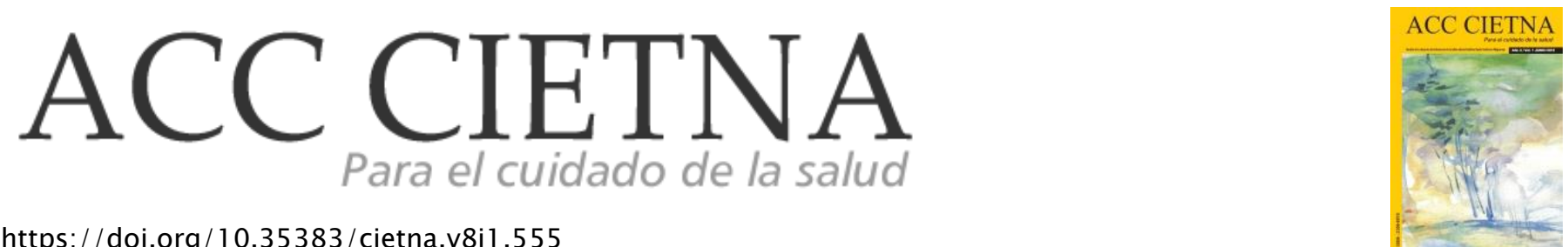

https:// doi.org/10.35383/cietna.v8i1 1.555

\title{
Diferencias en el puntaje del examen nacional de enfermería en postulantes al servicio rural y urbano marginal de salud
}

\author{
Asenjo Alarcón José Ander
}

INFORMACIÓN DEL ARTÍCULO RESUMEN

\section{Historia del artículo:}

Recibido el 06 de febrero de 2021

Aceptado el 09 de junio de 2021

\section{Palabras clave:}

Personal de enfermería (D009740)

Enfermería (Q000451)

Atención de enfermería (D009732)

Rol de la enfermera (D024802)

Rendimiento académico (D000073978)

Servicios de salud (D006296)
Objetivo: Determinar las diferencias en el puntaje del Examen Nacional de Enfermería en postulantes al Servicio Rural y Urbano Marginal de Salud en Cajamarca Perú, según universidad de procedencia. Método: Estudio observacional, transversal, retrospectivo, realizado en 68 postulantes al Servicio Rural y Urbano Marginal de Salud 2020 - I en enfermería, se utilizó la base de datos online del Ministerio de Salud. Resultados principales: El puntaje promedio obtenido por los postulantes de la Universidad Nacional Autónoma de Chota fue de $14,36 \pm 1,09$, de la Universidad Nacional de Cajamarca de 13,65 \pm 1,40, de la Universidad Privada de Chiclayo de 12,13 $\pm 0,64$, de la Universidad Privada San Pedro de 12,10 $\pm 0,84$, de la Universidad Privada Antonio Guillermo Urrelo de 11,98 $\pm 0,70$ y de la Universidad Alas Peruanas de 11,15 $\pm 0,21$. La diferencia global del puntaje entre universidades fue significativa $(p=0,000)$. Conclusión general: Comparando el puntaje obtenido entre pares de universidades, la Universidad Nacional Autónoma de Chota con la Universidad Nacional de Cajamarca no presentan diferencias significativas; pero si existen diferencias significativas entre la Universidad Nacional Autónoma de Chota y la Universidad Privada de Chiclayo, Universidad Privada San Pedro, Universidad Privada Antonio Guillermo Urrelo y Universidad Alas Peruanas.

'Doctor en Ciencias de la Salud. Docente de la Universidad Nacional Autónoma de Chota, Cajamarca, Perú. Email: ander1213@hotmail.com. ORCID: https://orcid.org/0000-0002-1059-4258 
Differences in the score of the national nursing exam in applicants to the rural and urban marginal health service

ABSTRACT

Keywords:

Nursing staff (D009740)

Nursing (Q000451)

Nursing care (D009732)

Nurse's role (D024802)

Academic performance (D000073978)

Health services (D006296)
Objective: to determine the differences in the score of the National Nursing Exam in applicants to the Rural and Urban Marginal Health Service in Cajamarca Peru, according to the university of origin. Method: Observational, cross-sectional, retrospective study, carried out in 68 applicants to the Rural and Urban Marginal Health Service 2020 - I in nursing, using the online database of the Ministry of Health. Main results: The average score obtained by the applicants of the National Autonomous University of Chota was $14.36 \pm 1.09$ of the National University of Cajamarca of $13.65 \pm 1.40$ of the Private University of Chiclayo of $12.13 \pm 0.64$ of the San Pedro Private University of $12.10 \pm$ 0.84 , of the Antonio Guillermo Urrelo Private University of $11.98 \pm 0.70$ and from the Alas Peruanas University of $11.15 \pm 0.21$. The overall difference in score between universities was significant $(p=0.000)$. General conclusion: Comparing the score obtained between pairs of universities, the National Autonomous University of Chota with the National University of Cajamarca do not present significant differences; but there are significant differences between the National Autonomous University of Chota and the Private University of Chiclayo, San Pedro Private University, Antonio Guillermo Urrelo Private University and Alas Peruanas University.

\section{Introducción}

El Servicio Rural y Urbano Marginal de Salud es realizado por los profesionales pertenecientes a las ciencias de la salud, una vez obtenido su título profesional y colegiatura; es un requisito primordial para que puedan desempeñarse en instituciones públicas. La Ley $\mathrm{N}^{\circ} 23330$, Ley del Servicio Rural y Urbano Marginal de Salud establece que el Ministerio de Salud como ente rector y juntamente con organismos públicos y privados de salud, son los responsables de la organización, planificación y ejecución del Servicio Rural y Urbano Marginal de Salud (1).

El Servicio Rural y Urbano Marginal de Salud debe realizarse preferentemente en puestos y centros de salud del primer nivel de atención (2), por ser establecimientos con mayor demanda de usuarios $y$ en los que existe carencia de profesionales estables, para responder a las demandas de la población. Como incentivo para los profesionales de salud en el año 2020, se está otorgando una bonificación según el grado de dificultad en el que se encuentra ubicado el establecimiento, para una postulación futura a cargos asistenciales, que va desde una bonificación de 1\% (grado de dificultad 1) hasta el 15\% (grado de dificultad 5) (3).

El licenciado en enfermería es uno de los profesionales que pertenecen a las ciencias de la salud, $y$ es el encargado de interactuar directamente con la población a través de actividades preventivo-promocionales, asistenciales, administrativas, educativas e investigativas (2), expresando las competencias adquiridas durante su formación profesional, en beneficio de la colectividad.

Para acceder al Servicio Rural y Urbano Marginal de Salud el profesional de enfermería debe rendir y 
aprobar un examen nacional, instaurado desde el año 2009 por la Asociación Peruana de Facultades y Escuelas de Enfermería (4), se realiza dos veces al año, el Examen Nacional de Enfermería ordinario y el Examen Nacional de Enfermería extraordinario; el examen consta de 100 preguntas y evalúa los conocimientos adquiridos por el personal de enfermería durante su formación profesional y su preparación para responder a sucesos futuros que enfrentará durante el Servicio Rural y Urbano Marginal de Salud. Las áreas que contempla el Examen Nacional de Enfermería son: Salud de la mujer, niño y adolescente $40 \%$, salud del adulto $30 \%$, enfermería fundamental o básica $10 \%$, salud pública $10 \%$, investigación en enfermería $5 \%$ y gerencia en salud $5 \%$ (5).

Las brechas de competencias alcanzadas por el profesional de enfermería son menores, en relación al profesional médico y obstetra, según la evaluación de competencias realizada por el Ministerio de Salud en las regiones La Libertad, Loreto y Piura; profesional de enfermería competente $95 \%$, profesional obstetra competente $84 \%$ y profesional médico competente $8 \%$, estando los porcentajes restantes en proceso de adquirir la competencia (6); no obstante, el trabajo de fortalecimiento de competencias debe continuarse y mejorarse, para que la respuesta ante las necesidades de la población sea oportuna y adecuada.

Las competencias adquiridas por el personal de enfermería, le permitirán desenvolverse eficientemente en diferentes contextos de desempeño, el grado de competencia alcanzado por el enfermero depende en gran medida de las exigencias y rigurosidad de la universidad durante la formación académica; en este sentido, analizar las competencias académicas de los postulantes al Servicio Rural y Urbano Marginal de Salud, es de suma importancia para plantear propuestas de mejora y mecanismos de retroalimentación en la formación profesional, y fortalecer aquellas competencias deficientes, lo que justifica el desarrollo de la investigación.
En el estudio se formuló la pregunta: ¿cuáles son las diferencias en el puntaje del Examen Nacional de Enfermería en postulantes al Servicio Rural y Urbano Marginal de Salud en Cajamarca según universidad de procedencia? y el objetivo fue determinar las diferencias en el puntaje del Examen Nacional de Enfermería en postulantes al Servicio Rural y Urbano Marginal de Salud en Cajamarca, según universidad de procedencia.

\section{Metodología}

\section{Diseño y población de estudio}

Estudio observacional, transversal, retrospectivo, realizado durante los meses de agosto y setiembre del año 2020. La población estudiada fue de 68 postulantes al Servicio Rural y Urbano Marginal de Salud 2020 - I en enfermería en la región Cajamarca Perú, procedentes de seis universidades: Universidad Nacional Autónoma de Chota, Universidad Nacional de Cajamarca, Universidad Privada de Chiclayo, Universidad Privada San Pedro, Universidad Privada Antonio Guillermo Urrelo y Universidad Alas Peruanas. Se incluyeron a todos los postulantes que rindieron el Examen Nacional de Enfermería.

\section{Medición de variables}

La técnica de recolección de datos fue el análisis documental de los puntajes alcanzados en el Examen Nacional de Enfermería por cada postulante. Los puntajes obtenidos en el Examen Nacional de Enfermería por cada postulante están disponibles en una base de datos online del Ministerio de Salud (7) y son de acceso público. En el proceso de Servicio Rural y Urbano Marginal de Salud 2020 - I, sólo fue considerado el puntaje obtenido en el Examen Nacional de Enfermería para la adjudicación de plazas ofertadas, a diferencia de procesos anteriores en que se sumaba con el promedio ponderado promocional de la universidad, teniendo un peso de $70 \%$ el 
Examen Nacional de Enfermería y $30 \%$ el promedio ponderado promocional (5).

\section{Procedimiento de recolección de datos}

Los datos se recolectaron a partir de la base online (en formato Excel) disponible por el Ministerio de Salud (7), se descargó el archivo para realizar el procesamiento respectivo.

\section{Análisis de datos}

La información necesaria se ingresó en una matriz de datos creada en el software estadístico SPSS v. 26 para Windows. El análisis estadístico univariado se realizó utilizando medidas de tendencia central y medidas de dispersión. El análisis estadístico bivariado se realizó mediante el ANOVA y la prueba post hoc de Scheffe, siendo estadísticamente significativo un $\mathrm{p}$-valor $<0,05$; previa demostración de normalidad con la prueba de Shapiro-Wilk y homocedasticidad con la prueba de Levene.

\section{Consideraciones éticas}

Los principios éticos, así como la conducta responsable en investigación, se mantuvieron durante todo el proceso investigativo. La identificación de cada postulante se realizó mediante un código para preservar la confidencialidad.

\section{Resultados, análisis y discusión}

El estudio se realizó con 68 postulantes al Servicio Rural y Urbano Marginal de Salud 2020 - I en enfermería para la región Cajamarca. El puntaje promedio obtenido por los postulantes de la Universidad Nacional Autónoma de Chota fue de 14,36 $\pm 1,09$, de la Universidad Nacional de Cajamarca de 13,65 $\pm 1,40$, de la Universidad Privada de Chiclayo de 12,13 $\pm 0,64$, de la Universidad Privada San Pedro de 12,10 0,84, de la Universidad Privada Antonio Guillermo Urrelo de $11,98 \pm 0,70$ y de la Universidad Alas Peruanas de $11,15 \pm 0,21$ (tabla 1).
Tabla 1. Puntaje del Examen Nacional de Enfermería en postulantes al Servicio Rural y Urbano Marginal de Salud en Cajamarca, según universidad de procedencia

\begin{tabular}{ccccc}
\hline \multirow{2}{*}{ Universidad } & $\mathbf{n}(68)$ & $\begin{array}{c}\text { Mín }- \\
\text { Máx }\end{array}$ & $\begin{array}{c}\text { Media } \pm \\
\text { D.S. }\end{array}$ & IC 95\% \\
\hline \multirow{2}{*}{ UNACH } & 12 & $12,3-$ & $14,36 \pm$ & 13,$67 ;$ \\
& & 16,2 & 1,09 & 15,05 \\
UNC & 31 & $11,3-$ & $13,65 \pm$ & 13,$14 ;$ \\
& & 17,2 & 1,40 & 14,17 \\
UPCH & 7 & $11,2-$ & $12,13 \pm$ & 11,$53 ;$ \\
& & 12,8 & 0,64 & 12,72 \\
UPSP & 7 & $11,1-$ & $12,10 \pm$ & 11,$32 ;$ \\
& & 11,3 & 0,84 & 12,88 \\
UPAGU & 9 & $13,4-$ & $11,98 \pm$ & 11,$44 ;$ \\
& & $11,0-$ & $11,15 \pm$ & 9,$24 ;$ \\
UAP & 2 & 11,3 & 0,21 & 13,06 \\
\hline UAP= Universidad & Alas &
\end{tabular}

$\mathrm{UAP}=$ Universidad Alas Peruanas, $\mathrm{UNACH}=$ Universidad Nacional Autónoma de Chota, UPSP = Universidad Privada San Pedro, UNC = Universidad Nacional de Cajamarca, UPAGU $=$ Universidad Privada Antonio Guillermo Urrelo, $\mathrm{UPCH}=$ Universidad Privada de Chiclayo.

La diferencia global del puntaje entre universidades fue significativa $(p=0,000)$ (tabla 2$)$. Comparando el puntaje obtenido entre pares de universidades, la Universidad Nacional Autónoma de Chota con la Universidad Nacional de Cajamarca no presentan diferencias significativas ( $p>0,05)$; pero si existen diferencias significativas entre la Universidad Nacional Autónoma de Chota y la Universidad Privada de Chiclayo, Universidad Privada San Pedro, Universidad Privada Antonio Guillermo Urrelo y Universidad Alas Peruanas ( $p<$ 0,05) (tabla 3). 
Tabla 2. Diferencias en el puntaje del Examen Nacional de Enfermería en postulantes al Servicio Rural y Urbano Marginal de Salud en Cajamarca, según universidad de procedencia

\begin{tabular}{llllll}
\hline Puntaje & $\begin{array}{l}\text { Suma de } \\
\text { cuadrados }\end{array}$ & gl & $\begin{array}{l}\text { Media } \\
\text { cuadrática }\end{array}$ & F & $\begin{array}{l}\text { p- } \\
\text { valor }\end{array}$ \\
\hline $\begin{array}{l}\text { Entre } \\
\text { grupos }\end{array}$ & 60,694 & 5 & 12,139 & 9,100 & $0,000^{*}$ \\
$\begin{array}{l}\text { Dentro } \\
\text { de }\end{array}$ & 82,701 & 62 & 1,334 & & \\
grupos & & & & & \\
\hline Total & 143,395 & 67 & & & \\
\hline${ }^{*} p<0,05$. & & & &
\end{tabular}

Tabla 3. Prueba post hoc para las diferencias en el puntaje del Examen Nacional de Enfermería en postulantes al Servicio Rural y Urbano Marginal de Salud en Cajamarca, según universidad de procedencia

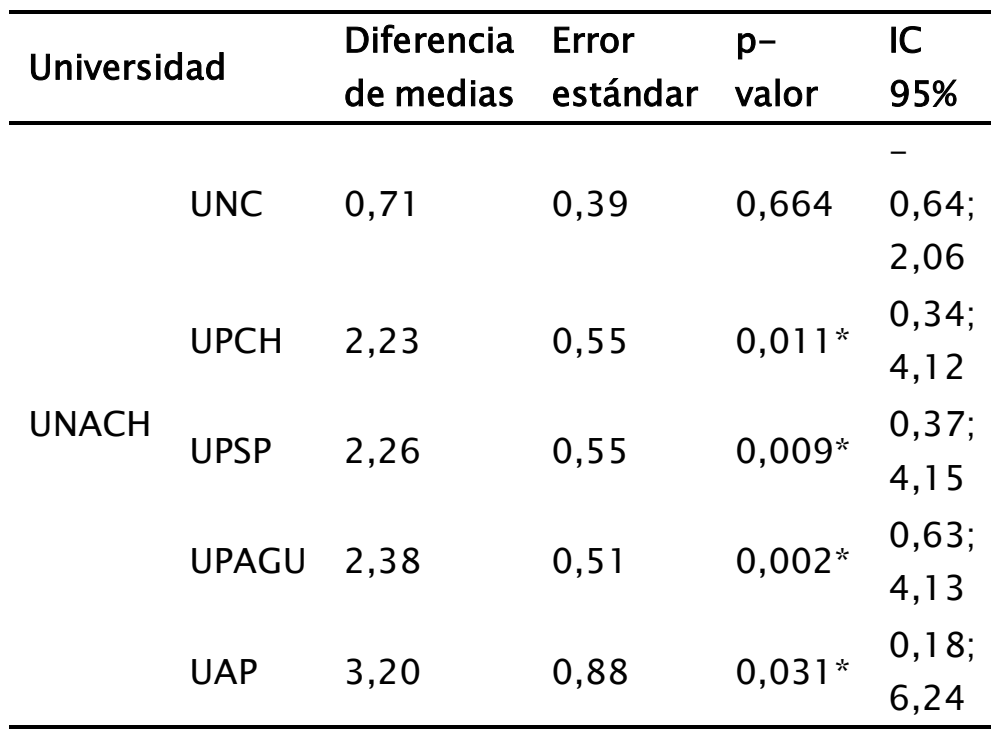

$\mathrm{UAP}=$ Universidad Alas Peruanas, $\mathrm{UNACH}=$ Universidad Nacional Autónoma de Chota, UPSP $=$ Universidad Privada San Pedro, UNC = Universidad Nacional de Cajamarca, UPAGU $=$ Universidad Privada Antonio Guillermo Urrelo, $\mathrm{UPCH}=$ Universidad Privada de Chiclayo.

*Prueba de Scheffe (La diferencia de medias es significativa en el nivel 0,05).

En términos generales, los postulantes egresados de las universidades nacionales alcanzaron mayor puntaje en relación a los postulantes de las universidades privadas. Así mismo, los postulantes de la Universidad Nacional Autónoma de Chota alcanzaron un puntaje promedio superior a los postulantes de la Universidad Nacional de Cajamarca. La Universidad Nacional Autónoma de Chota es una universidad de reciente creación y los postulantes al Servicio Rural y Urbano Marginal de Salud constituyen la primera promoción de egresados.

Las universidades nacionales se han caracterizado por tener mayores exigencias y rigurosidad para sus condiscípulos en el proceso de formación académica y esto se reflejaría a la hora de responder un examen y en su desenvolvimiento en contextos reales de trabajo. Las exigencias son notorias desde el proceso de admisión a la universidad, pues tienen que competir con otros postulantes para poder ingresar $y$ en muchos casos alcanzar un puntaje mínimo.

En las últimas décadas, la expansión de las universidades privadas en el Perú ha sido acelerada, junto a ellas las facultades y escuelas de enfermería; la mayoría fueron creadas con fines de lucro, dejando de lado la misión real de la universidad: la formación integral de los futuros profesionales con estándares de calidad en las competencias y perfiles alcanzados. La percepción del estudiante en una universidad privada es que, al pagar una mensualidad por su formación académica, los docentes están obligados a aprobarlos, incluso sin haber logrado las capacidades y competencias de las áreas académicas. El resultado de esto es una escasa o nula exigencia del futuro profesional para ser promotor de su propio aprendizaje, desvalorando la adquisición permanente de conocimiento y práctica que caracteriza al profesional de enfermería (8).

Recientemente, desde el año 2019 existe un proceso de regulación para la creación de facultades y escuelas profesionales, estableciéndose lineamientos para tal fin por parte de la Superintendencia Nacional de Educación Superior Universitaria, que aplica para universidades públicas y privadas, entre ellos: 
demanda social, disponibilidad de recursos, contextualización de la oferta educativa y estructuración orgánica y funcional de la normativa académica en todos sus niveles (9). Así mismo, la Superintendencia Nacional de Educación Superior Universitaria ha establecido condiciones básicas de calidad que deben cumplir las universidades en general, para que sigan brindando el servicio educativo (10). Se espera que las reformas instauradas mejoren realmente la calidad académica universitaria, contribuyendo al logro eficiente de las competencias profesionales $(11,12)$.

Las diferencias entre los puntajes obtenidos en el Examen Nacional de Enfermería por postulantes de universidades nacionales y privadas son estadísticamente significativas, lo que confirmaría la mayor preparación de los primeros en torno a su formación académica. No obstante, los contextos reales de desempeño laboral serán los que marquen objetivamente las diferencias en las competencias logradas por cada profesional, las que deben fortalecerse constantemente.

Adicionalmente, las características de los individuos también podrían tener una injerencia importante en el rendimiento profesional, independientemente de la universidad de procedencia; dado que, la flexibilidad a la adaptación a situaciones diversas contribuirá en gran medida al éxito profesional, pues estudiar en una universidad privada no repercute significativamente en el ámbito de desempeño profesional ni en la percepción pecuniaria (13).

Las universidades tanto públicas como privadas deben apuntar hacia el mismo horizonte; es decir, fortalecer las competencias de los profesionales de enfermería durante su formación académica debe ser el punto de intersección entre ambas, pues en último término el que se beneficiará será el usuario atendido en los distintos niveles de atención del sistema de salud. Por ello, las universidades deben establecer alianzas estratégicas en pro de un trabajo coordinado y planificado de beneficio común (14), para la formación de profesionales íntegros, con calidad humana, sentido común, valores y principios, que contribuyan al desarrollo del país (15), como lo indica la ley universitaria.

El profesional de enfermería constituye la parte medular del sistema de salud, representa el $59 \%$ de la fuerza de trabajo en la Región de las Américas, siendo el 38\% menores de 35 años, se gradúan 81,2 profesionales de enfermería por cada 100 000 habitantes por año (16). En el Perú existen 15,6 enfermeros por cada 10000 habitantes y en Cajamarca 12,6 (17), pasando en este último caso de 1176 personas por enfermero en el 2009 a 696 en el 2017 (18); sin embargo, aún es insuficiente. La coyuntura actual por la pandemia de covid-19 ha permitido vislumbrar la carencia de estos profesionales en los distintos niveles de atención. Siendo un pilar fundamental en el sistema de salud, debe estar preparado para responder a las necesidades de la población, con conocimientos, liderazgo y toma adecuada de decisiones, sobre todo en el primer nivel de atención (19,20,21,22), donde inicialmente se desempeña como serumista.

Las limitaciones del estudio fueron: el Examen Nacional de Enfermería no es suficiente para evaluar de forma integral las competencias adquiridas por el profesional de enfermería; el resultado del Examen Nacional de Enfermería no se publica por áreas del conocimiento de enfermería, que permita analizar las competencias $y$ capacidades que deben ser fortalecidas en la formación académica.

\section{Conclusiones}

En conclusión, el puntaje promedio obtenido por los postulantes en orden descendente corresponde a la Universidad Nacional Autónoma de Chota con 14,36, Universidad Nacional de Cajamarca con 13,65, Universidad Privada de Chiclayo con 12,13, Universidad Privada San Pedro con 12,10, Universidad Privada Antonio Guillermo Urrelo con 11,98 y Universidad Alas Peruanas con 11,15. La diferencia global del puntaje entre universidades fue significativa. Finalmente, la Universidad Nacional Autónoma de Chota y la Universidad 
Nacional de Cajamarca no presentan diferencias significativas en el puntaje obtenido; pero si existen diferencias significativas entre la Universidad Nacional Autónoma de Chota y la Universidad Privada de Chiclayo, Universidad Privada San Pedro, Universidad Privada Antonio Guillermo Urrelo y Universidad Alas Peruanas.

Las diferencias en el puntaje obtenido por los postulantes representan un insumo importante para que las universidades tanto públicas como privadas uniformicen criterios con metas comunes en la formación profesional de enfermería, con la finalidad de reducir las brechas en el logro de competencias, mediante un trabajo integral $y$ compartido. Así mismo, deben fortalecer el desarrollo de habilidades blandas en los futuros enfermeros para mejorar su capacidad de resiliencia, que le servirá de mucho frente a situaciones adversas.

\section{Bibliografía}

1. Ley del Servicio Rural y Urbano Marginal de Salud. Ley $\mathrm{N}^{\circ} 23330 / 1981$ de 10 de diciembre [internet]. Diario Oficial El Peruano, (11-121981) [Fecha de acceso: 15 Agos 2020]. Disponible en:

https://cdn.www.gob.pe/uploads/document/fi le/520483/ley-del-servicio-rural-y-urbanomarginal-de-salud-ley-n-23330.pdf

2. Reglamento de la Ley $N^{\circ} 23330$, Servicio Rural y Urbano Marginal de Salud - SERUMS. Decreto Supremo N 005-97-SA/1997 de 20 de junio [internet]. Diario Oficial El Peruano, (22-061997) [Fecha de acceso: 15 Agos 2020]. Disponible en: http://www.cbperu.org.pe/wpcontent/uploads/2019/07/10.-D.S.N\%C2\%B0-005-97-SA-aprueba-el-

Reglamento-de-la-ley-N\%C2\%BO-

23330-\%E2\%80\%9CLey-del-Servicio-Rural-yUrbano-marginal-de-Salud-\%E2\%80\%93SERUMS..pdf

3. Ministerio de Salud. Resolución Ministerial $\mathrm{N}^{\circ}$ 258-2020-MINSA, modificación de la R.M. N ${ }^{\circ}$ 215-2020-MINSA y el Reglamento de la Ley del
SERUMS [internet]. Lima; 2020 [Fecha de acceso: 14 Ago 2020]. Disponible en:

https://busquedas.elperuano.pe/normaslegale s/modifican-la-rm-n-215-2020-minsa-y-elreglamento-de-la-le-resolucion-ministerialn-258-2020-minsa-1866086-1/

4. Ministerio de Salud. Resolución Ministerial $N^{\circ}$ 575-2009-MINSA, Establecen disposiciones para la realización del Examen Nacional de Enfermería - ENAE [internet]. Lima; 2009 [Fecha de acceso: 14 Agos 2020]. Disponible en: https://www.gob.pe/institucion/minsa/norma s-legales/246319-575-2009-minsa

5. Asociación Peruana de Facultades y Escuelas de Enfermería. Reglamento del Examen Nacional de Enfermería (modalidad no presencial) [internet]. Lima; 2020 [Fecha de acceso: 1 Oct 2020]. 13 p. Disponible en:

https://enae.aspefeen.org.pe/doc/100\%20pre guntas\%20-\%20REGLAMENTO\%20FINAL\%20EN AE\%20NO\%20PRESENCIAL-01-09-2020.pdf

6. Ministerio de Salud. Evaluación de competencias a los profesionales médicos, obstetras y enfermeros del Servicio Rural y Urbano Marginal en Salud SERUMS [internet]. Lima; 2018 [Fecha de acceso: 1 Oct 2020]. 82 p. Disponible

en: http://bvs.minsa.gob.pe/local/MINSA/4337.pd f

7. Ministerio de Salud. Listado final de aptos al proceso SERUMS 2020 - I (remunerados y equivalentes a nivel nacional) [internet]. Lima; 2020 [Fecha de acceso: 10 Agos 2020]. 2 p. Disponible en:

https://www.gob.pe/institucion/minsa/inform es-publicaciones /663143-listado-final-deaptos-al-proceso-serums-2020-iremunerados-y-equivalentes-a-nivel-nacional

8. Benavides M, León J, Haag F, Cueva S. Expansión y diversificación de la educación superior universitaria, y su relación con la desigualdad y la segregación [internet]. Lima; 2015 [Fecha de acceso: 1 Oct 2020]. 76 p. Disponible en:

https://www.grade.org.pe/wpcontent/uploads/ddt78.pdf 
9. Superintendencia Nacional de Educación Superior Universitaria. Resolución del Consejo Directivo $\quad N^{\circ}$ 066-2019-SUNEDU/CD, Estándares para la creación de facultades y escuelas profesionales [internet]. Lima; 2019 [Fecha de acceso: 12 Oct 2020]. Disponible en: https://intranet.sunedu.gob.pe/documentos/d irectorios/320/res-066-2019-sunedu-cdresuelve-aprobar-los-estandares-para-lacreacion-de-facultades-y-escuelas.pdf

10. Superintendencia Nacional de Educación Superior Universitaria. Condiciones básicas de calidad [internet]. Lima; 2016 [fecha de acceso 12 de octubre de 2020]. Disponible en: https://www.sunedu.gob.pe/8-condicionesbasicas-de-calidad/

11. Cuenca R. La educación universitaria en el Perú Democracia, expansión y desigualdades. [internet]. Lima; 2015 [Fecha de acceso: 2 Nov 2020]. 160 p. Disponible en: https://repositorio.iep.org.pe/bitstream/IEP/6 $\underline{03 / 2 / \text { estudiossobredesigualdad 10.pdf }}$

12. British Council. La reforma del sistema universitario peruano: Internacionalización, avance, retos y oportunidades. [internet]. Lima; 2016 [Fecha de acceso: 2 Nov 2020]. 130 p. Disponible en: https://docplayer.es/66292446-La-reformadel-sistema-universitario-peruanointernacionalizacion-avance-retos- $y$ oportunidades.html

13. Canal JF y Rodríguez C. Universidad pública frente a universidad privada: ¿qué efectos tiene sobre el éxito profesional de los universitarios españoles? Rev. Esp. Investig. Sociol [internet]. 2020 [Fecha de acceso: 2 Nov 2020]; 169: 21 $40 . \quad$ Disponible en: http://www.reis.cis.es/REIS/PDF/REIS_169_021 579004489856.pdf

14. Dibbern A, Sampaio $H$, Lemaitre $M J$, Takayanagui AD, Albornoz O. La educación superior pública y privada en América Latina y el Caribe. Contexto de internacionalización y proyecciones de políticas públicas [internet]. París; 2016 [Fecha de acceso: 2 Nov 2020]. 193 p. Disponible en: https://www.ses.unam.mx/curso2016/pdf/11 -nov-IESALC.pdf

15. Ley Universitaria. Ley $\mathrm{N}^{\circ} 30220 / 2014$ de 8 de julio [internet]. Diario Oficial El Peruano, (9-72014) [Fecha de acceso: 15 Agos 2020]. Disponible

en:

https://diariooficial.elperuano.pe/pdf/0021/le y-universitaria-30220.pdf

16. De Bortoli SH, Munar EF, Umpiérrez A, Peduzzi M, Leija C. La situación de la enfermería en el mundo y la Región de las Américas en tiempos de la pandemia de COVID-19. Rev Panam Salud Publica [internet]. 2020 [Fecha de acceso: 15 Agos 2020]; 44: e64. Disponible en: https://doi.org/10.26633/RPSP.2020.64

17. Sociedad de Comercio Exterior del Perú. Rumbo al bicentenario: Recursos humanos de salud [internet]. Lima; 2020 [Fecha de acceso: 2 Nov 2020]. 4 p. Disponible en: https://www.comexperu.org.pe/articulo/rumb o-al-bicentenario-recursos-humanos-desalud

18. Instituto Nacional de Estadística e Informática. Número de habitantes por cada enfermera/o, según departamento, 2009-2017 [internet]. Lima; 2019 [Fecha de acceso: 2 Nov 2020]. 1 p. Disponible en: https://www.inei.gob.pe/estadisticas/indicetematico/health-human-resources/

19. Organización Panamericana de la Salud. Ampliación del rol de las enfermeras y enfermeros en la atención primaria de salud [internet]. Washington, D.C.; 2018 [Fecha de acceso: 2 Nov 2020]. 54 p. Disponible en: https://iris.paho.org/bitstream/handle/10665. 2/34959/9789275320037_spa.pdf?sequence $=$ 5\&isAllowed $=y$

20. Organización Mundial de la Salud. La OMS y sus asociados hacen un llamamiento urgente para que se invierta en el personal de enfermería [internet]. Ginebra; 2020 [Fecha de acceso: 2 de noviembre de 2020]. 4 p. Disponible en: https://www.who.int/es/newsroom/detail/07-04-2020-who-and-partnerscall-for-urgent-investment-in-nurses 
21. Elsevier Connect. Año 2020: El mundo rinde homenaje a los profesionales de Enfermería. [internet]. Ámsterdam; 2020 [Fecha de acceso: 8 Dic 2020]. 10 p. Disponible en:

https://www.elsevier.com/eses/connect/enfermeria/ano-2020-el-mundorinde-homenaje-a-los-profesionales-deenfermeria

22. Echevarría P. La disciplina enfermera solo puede crecer a través de la investigación. [internet]. Ámsterdam; 2020 [Fecha de acceso: 8 Dic 2020]. Elsevier Connect. 10 p. Disponible en:

https://www.elsevier.com/es-

es/connect/enfermeria/paloma-echevarria-ladisciplina-enfermera-solo-puede-crecer-atraves-de-la-investigacion 